\title{
XXXI. Ueber das Vorkommen von Astrophyllit, Arfvedsonit und Zirkon in El Paso Co. Colorado.
}

Von

Georg August König in Philadelphia.

Vorkommen im Allgemeinen. Die genannten Mineralien liegen in massivem, grauem Quarz eingebettet, und zwar so, dass ań den mir bis jetzt vorliegenden Handstucken Astrophyllit und Zirkon unzertrennlich erscheinen, während der Arfvedsonit ganz isolirt im Quarze vorkommt. Jedoch muss eine genaue Untersuchung an Ort und Stelle angestellt werden, um uber die Stetigkeit dieser Association ins Klare zu gelangen, was bisher noch nicht geschehen ist. Andere Handstucke, ganz vom Aussehen der norwegischen, zeigen den Astrophyllit eingewachsen in Orthoklas. Das Muttergestein scheint demnach auch hier Syenit zu sein und der Astrophyllit hauptsächlich in quarzreichen Ausscheidungen desselben vorzukommen. An Stùcken von der Oberfläche ist der Quarz fast glanzlos und häufig durch Eisenocker gebräunt, während in einiger Teufe der Glanz sehr ausgeprägt wird und ockerige Zersetzungsproducte ganz fehlen. So viel mir bekannt, ist Astrophyllit bis jetzt nur im Syenit von Brevig in Norwegen vorgekommen und auch dort mit Arfvedsonit und Zirkon vergesellschaftet, jedoch nur in fleischrothem Feldspathe eingesprengt. Die Parallele erschien mir so interessant, dass ich mich veranlasst fublte, die Mineralien des neuen Fundortes genauer zu studiren.

Dem Herrn Dr. med. E. F o ote bin ich für Ueberlassung seines gesammten Materiales zu grossem Danke verpflichtet.

\section{Astrophyllit.}

Die Krystalle erscheinen als sehr in die Länge gestreckte, meistens dünne und schmale Säulen, deren oft parallele Anordnung einen schilfartigen Habitus des Gesteins bedingt. Jedoch ist die gegenseitige Lage ebenso häufig eine durchaus regellose und verworrene. Nach dem Herauslösen der Krystalle hinterlassen sie ein ausserordentlich scharfes Negativ 
im Quarze, so zwar, dass die feinste Streifung deutlich hervortritt. Im Querschnitte zeigen die Krystalle scheinbar ein Rechteck, das an einigen Krystallen deutlich noch schiefe Abstumpfungsflächen zeigt. Terminale Flächen sind durchaus nicht vorhanden; die Individuen erscheinen durchweg durch einen Bruch begrenzt, oder unmerklich in den Quarz verlaufend. Die in der Nachschrift mitgetheilte optische Untersuchung des Herrn Dr. H. B u ck ing beweist, dass die Krystalle dem m o n o s ymmetri s chen Systeme angehören, und dass die Längsrichtung der Symmetrieebene entspricht. Wählt man die Ebene der vollkom-

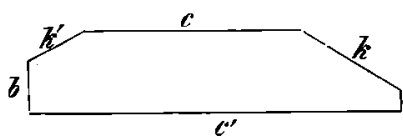
menen Spaltbarkeit zur Basis, so sind demnach die Krystalle nach der Klinodiagonale verlängert. Der senkrecht zu dieser Axe stehende Querschnitt zeigte an dem bestentwickelten Krystall die in beistehender Figur dargestellte Form, an welcher folgende Winkel mit dem Reflexionsgoniometer annähernd gemessen werden konnten:

$$
\begin{array}{lrl}
c: c^{\prime}=4^{0} & c: k=30 \frac{1}{2}{ }^{\circ} \\
b: c^{\prime}=\mathbf{8 7 0}^{0} & c: k^{\prime}=32^{0} \\
b: c=9^{0} & b: k^{\prime}=61^{0}
\end{array}
$$

Von diesen Flächen war $c^{\prime}$ eine Spaltungsfläche und spiegelte scharf, die ubrigen, welche mit Ausnahme von $c$ nur sehr unvollkommen Licht reflectirten, waren natüliche Krystallfächen. Da bei letzteren die Unsicherheit der Einstellung $1{ }^{0}$ betrug, so ist es wahrscheinlich, dass der Winkel $c: k$ und $c: k^{\prime}$ als gleich zu betrachten sei und $k k^{\prime}$ demnach ein Klinodoma vorstelle, dagegen ist es unwahrscheinlich, dass $b: c$ als rechter Winkel und $b$ als Symmetrieebene aufzufassen sei; wahrscheinlich ist letztere Fläche ein nur einseitig entwickeltes, sehr steiles Klinodoma. Alle Krystallflächen sind parallel der Klinodiagonale stark gestreift.

Die Spaltbarkeit nach $c$ ist eine sehr vollkommene, ein zweiter erkennbarer Bruch ist vorhanden, und zwar nach der Ebene des oben gezeichneten Querschnittes, also wahrscheinlich nach dem Orthopinakoid. Das Mineral ist spröde und lässt sich im Mörser leicht sè fein pulvern. Die Härte auf der Spaltungsfläche beträgt ungefähr 3.

Die Farbe auf den glänzenden $c$-Flächen ist messinggelb bis broncebraun; wegen des fast halbmetallischen Glanzes erscheint das Pulver bei einer gewissen Feinheit dem Mussivgolde ähnlich. Im durchfallenden Lichte sind die Spaltungsplättchen, je nach ihrer Dicke, gelb bis tief rothbraun (die weitere optische Untersuchung siehe am Schluss dieses Aufsatzes).

Volumgewicht $=3,375$ bei $45 \mathrm{G} 0$.

Das Mineral schmilzt vor dem Löthrohre leicht zur schwarzen Kugel, wobei es ein wenig sich aufbläht. Mit Phosphorsalz erhält man leicht die 
Reactionen auf Kieselsäure, Eisen, Mangan und Titan, schwieriger die Flammenfärbung von Kalium. Im Kölbchen giebt es etwas Wasser. Salzund Schwefelsäure zersetzcn dis Pulver leicht unter dem Kochpunkte, und in der zugeschmolzenen Röhre bei $140 \mathrm{C}^{0}$ erfolgt vollständiges Aufschliessen in kurzer Zeit.

\begin{tabular}{|c|c|c|c|c|}
\hline \multicolumn{5}{|c|}{ Zusammensetzung } \\
\hline $\mathrm{SiO}_{2}$ & $=$ & 34,68 & 18,485 & \multirow{3}{*}{24,363} \\
\hline $\mathrm{TiO}_{2}$ & $=$ & 13,58 & 5,299 & \\
\hline $\mathrm{ZrO}_{2}$ & $=$ & 2,20 & 0,579 & \\
\hline $\mathrm{Fe}_{2} \mathrm{O}_{3}$ & $=$ & 6,56 & 1,968 & \multirow{2}{*}{2,294} \\
\hline $\mathrm{Al}_{2} \mathrm{O}_{3}$ & $=$ & 0,70 & 0,326 & \\
\hline $\mathrm{FeO}$ & $=$ & 26,10 & 5,799 & \multirow{6}{*}{8,256} \\
\hline $\mathrm{MnO}$ & $=$ & 3,48 & 0,784 & \\
\hline $\mathrm{K}_{2} \mathrm{O}$ & $=$ & 5,01 & 0,851 & \\
\hline $\mathrm{Na}_{2} \mathrm{O}$ & $=$ & 2,54 & 0,655 & \\
\hline $\mathrm{MgO}$ & $=$ & 0,30 & 0,120 & \\
\hline $\mathrm{CuO}$ & $=$ & 0,42 & 0,047 & \\
\hline $\mathrm{Ta}_{2} \mathrm{O}_{5}$ ? & $=$ & 0,80 & \multirow{3}{*}{$3,147\}$} & \multirow{3}{*}{3,147} \\
\hline \multirow[t]{2}{*}{$\mathrm{H}_{2} \mathrm{O}$} & & 3,54 & & \\
\hline & & 9,91 & & \\
\hline
\end{tabular}

Hieraus resultiren die Atomverhältnisse $\stackrel{\mathrm{VI}}{\mathrm{Fe}_{2}}=1$ gesetzt), welche in der weiter unten folgenden Tabelle angeführt sind; deren letzte Columne entbält die zu ganzen Zahlen abgerundeten Werthe, wobei zu bemerken ist, dass hierbei der Sauerstoff naturgemäss die grösste Correction erfahren muss, weil sich bei ihm die Fehler sämmtlicher Bestimmungen summiren. Zirkonium wurde in das gleichwerthige Titan verwandelt, Aluminium in sechswerthiges Eisen. Die Tantalsäure wurde nicht berucksichtigt, weil ich bei dem gegenwärtigen Stande unserer Kenntniss des Titans, Zirkoniums und auch der Tantalsäure selbst in ihrem Zusammenvorkommen mich ausser Stande sah, die létztere Säure als definitiv vorhanden zu erklären. Beim Schmelzen der durch Kochen gefällten Oxyde mit $\mathrm{KH} \mathrm{SO}_{4}$ und Lösen der Schmelze in kaltem Wasser blieb ein unlöslicher Ruckstand, der ganz das Aussehen des Tantalsulfates zeigte. Er löste sich in warmer Natronlauge und wurde durch Zusatz eines Ueberschusses von verdunnter Schwefelsäure in der Kälte abgeschieden, liess sich durch Fluorwasserstoffsäure nicht verfluchtigen und löste sich in Phosphorsalz zur ungefärbten Perle in der Kälte. Dieses Verhalten stimmt mit dem der Tantalsäure uberein. In meiner nun schon uber ein Jahr fortgesetzten Arbeit uber Titan und Zirkonium sind mir jedoch soviele, in der Literatur nicht berührte, Anomalien vorgekommen, dass ich gegenwärtig mich ausser Stande fühle zu sagen, was die genannten Metalle unter Umständen thun können 
oder nicht. Ich habe daher vorgezogen, trotz der scheinbaren Coincidenz der Reactionen, den geringen Betrag der Tantalsäure, mit einem Fragezeichen versehen, vorläufig in der Formel nicht zu berücksichtigen, da ja uberdies der allgemeine Ausdruck kaum merklich geändert wlirde.

\begin{tabular}{|c|c|c|c|c|c|c|c|c|}
\hline \multicolumn{9}{|c|}{ Atomverhältnisse $\left(\mathrm{Fe}_{2}=1\right.$ gesetzt $)$ : } \\
\hline$s i=$ & 16,195: & 28 & $=0,578$ & 13,13 & de & ab & eru & $=13$ \\
\hline$T i=$ & 9,281: & 50 & $=0,185$ & 4,25 & " & , & ” & 4 \\
\hline$F e_{2}=$ & $5,010:$ & 112 & $=0,044$ & 1,00 & $"$ & & " & 1 \\
\hline $\mathrm{Fe}=$ & $20,331:$ & 56 & $=0,363$ & 8,25 & 》 & & » & 8 \\
\hline$M n=$ & 2,696 & 55 & $=0,049$ & 1,11 & ) & & ) & 1 \\
\hline $\boldsymbol{K}=$ & $4,159:$ & 39 & $=0,107$ & 2,43 & & & & \\
\hline$N a=$ & 1,885: & 23 & $=0,081$ & 1,84 & ") & & 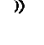 & 4 \\
\hline$=$ & 0,393 & 1 & $=0,393$ & 8,93 & ) & & " & 8 \\
\hline$=$ & $38,060:$ & 16 & $=2,379$ & 54,07 & » & & 》 & 52 \\
\hline
\end{tabular}

Unter der Annahme, dass der Gehalt an Wasser ein wenig zu hoch gefunden worden sei, würde hieraus die empirische Formel

$$
\mathrm{H}_{8}(\mathrm{~K}, \mathrm{Na})_{4}(\mathrm{Fe}, \mathrm{Mn})_{9} \stackrel{\mathrm{VI}}{\mathrm{FI}} \mathrm{Fi}_{2} \mathrm{Si}_{43} \mathrm{O}_{52}
$$

folgen, d. h. diejenige eines Singulosilikates (eines Salzes der Säure $\mathrm{H}_{4} \mathrm{SiO}_{4}$ ).

Vergleicht man nun meine Analyse mit denen von Pisani, Scheerer, Meinecke und Sieveking, welche den Astrophyllit von Brevig untersucht haben (Dana, Mineralogy. 5. ed. 309), so ergeben sich, neben allgemeiner Uebereinstimmung, doch im Einzelnen beträchtliche Differenzen.

Zur besseren Uebersicht sollen die Resultate der Analytiker hier neben einander gestellt werden :

\begin{tabular}{|c|c|c|c|c|c|}
\hline & Pisani & Scheerer & Meinecke & Sieveking & König \\
\hline $\mathrm{SiO}_{2}=$ & 33,23 & 32,21 & 32,35 & 33,71 & 34,68 \\
\hline $\mathrm{TiO}_{2}=$ & 7,09 & 8,24 & 8,84 & 8,76 & 13,58 \\
\hline $\mathrm{ZrO}_{2}=$ & 4,97 & - & - & - & $\mathbf{2 , 2 0}$ \\
\hline $\mathrm{Al}_{2} \mathrm{O}_{3}=$ & 4,00 & 3,02 & 3,64 & 3,47 & 0,70 \\
\hline$M n O=$ & 9,90 & 12,63 & 12,68 & 10,59 & 3,48 \\
\hline
\end{tabular}

Hinsichtlich der Kieselsäure herrscht, wie man sieht, durchaus eine befriedigende Uebereinstimmung. Addirt man Titansäure, Zirkonerde und Thonerde, so erhält man :

Pisani $=16,06 \quad$ Scheerer $=11,26 \quad$ Sieveking $=12,23$

König $=16,48 \quad$ Meinecke $=12,30$

Die ersten sind der Quantität nach gleich und nur der Qualităt nach verschieden. Die drei letzteren sind unter sich ubereinstimmend, von den ersten aber wesentlich abweichend. - Erinnert man sich nun der Sehwierigkeiten, welche von einer richtigen quantitativen Scheidung der drei Me- 
talle unzertrennlich sind, ja der zur Zeil bestehenden Unmöglichkeit einer solchen Trennung, so wird man nicht umhin können, die chemische Gleichartigkeit des Astrophyllites von Brevig und dessen von Color a do anzuerkennen. Hingegen bedingt der offenbar weil niedrigere Mangangehalt in dem letzteren Minerale eine spezifische Verschiedenheit. - Auffallend erscheint auch die Abwesenheit der Zirkonerde in den Analysen von Scheerer, Meinecke und Sieveking; deshalb ging ich in meiner Untersuchung möglichst kritisch zu Werke und bediente mich einer in der Literatur meines Wissens nicht erwähnten Methode, welche nachstehend den Fachgenossen zur Prufung bei ähnlichen Untersuchungen thergeben werden soll. -

Der engen Vergesellschaftung des Astrophyllites und Zirkones an dem neuen Fundorte wurde bereits fruher gedacht. Nachdem der Astrophyllit in kleinen Stückchen mit möglichster Sorgfalt, unter Zuhúlfenahme der Lupe, ausgelesen worden, nahm man beim Zerreiben des sonst weichen Materials im Mörser hin und wieder ein Knirschen wahr, welches offenbar von einem harten beigemengten Körper herruhrte. - Da das Mineral sich ohne Schwierigkeit durch Schwefelsäure zersetzen lässt, so vermied man beim Zerkleinern das Reiben, um die kleinen, harten Körner möglichst wenig zu zermalmen. - Die Zersetzung wurde nun durch 50procentige Schwefelsäure bewerkstelligt, und die abfiltrirte Kieselsäure mit Aelznatron digerirt. Dabei blieb ein weisser, körniger und harter Ruckstand im Betrag von 3 bis 6 Prozent in verschiedenen Proben. Der Ruckstand wurde für sich analysirt und erwies sich in der That als Zirkon, dem elwas Quarz beigemengt war. Da nun Zirkon als feinstes Pulver auch bei längerer Digestion mit Schwefelsäure kaum merklich zersetzt wird, so ist man in diesem Falle berechtigt, die in Lösung gegangene Zirkonerde als Bestandtheil des Astrophyllites zu betrachten. Die Kieselsäure wurde aus der alkalischen Lösung durch Ansäuren und Abdampfen abgeschieden und nach dem Wägen durch Fluorwasserstoffsäure verflüchtigt; der hierbei bleibende Rückstand (Titansäure) in Abrechnung gebracht.

Die schwefelsaure Lösung der Metalle wurde vorsichtig neutralisirt $\left(\mathrm{Na}_{2} \mathrm{CO}_{3}\right)$, mittelst Schwefelwasserstoff das Eisen in Oxydul übergeführt und nach Verdünnung auf $800 \mathrm{CC}$ durch Kochen der grösste Theil der Titansäure abgeschieden. Aus dem Filtrate schied man mit den bekannten Vorsichtsmassregeln die Sesquioxyde durch essigsaures Natron.

Diese, grösstentheils aus Eisenoxyd bestehend, wurden nach dem Glühen und Wägen im Wasserstoffstrome geglüht und dann durch Chlorgas der grösste Theil des Eisens verfluchtigt (es gelang mir nie das Eisen vollständig fortzutreiben). Der Rückstand wurde in Schwefelsäure und Fluorwasserstoffsäure gelöst, und die völlige Abscheidung des Eisens durch 
Schwefelammonium in Gegenwart von Ammoniumcitrat im Ueberschusse bewerkstelligt. Jer durch Abdampfen des Filtates und Einäschern gewonnene Rückstand wurde mit Wasser ausgekocht, um etwaige alkalische Salze zu entfernen und dann gewogen. Hierauf löste man denselben in einigen Tropfen Fluorwasserstoffsäure, dampfte mit Schwefelsäure ab bis nahezu zur Trockne und lösle in einem Minimum Wasser oder, wenn mehr gebraucht worden, dampfte man auf ein entsprechendes Volum, ca. 2 Cub.-C., ab, brachte die Fliussigkeit auf ein Uhrglas und setzte eine Menge von Ammonsulfat zu, welche hinreichen würde zur Alaunbildung, wenn die Summe der Oxyde Thonerde wäre. Man uberliess nun die Lösung der freiwilligen Verdunstung zur Trockne an einem mässig warmen Orle und brachte dann mit wenigen Tropfen kalten Wassers die Sulfate des Titans und Zirkoniums nebst dem Ueberschusse des Ammonsulfates in Lösung. Der gebildele Alaun ist so körnig, dass er sich durch Abschlämmen von der Mutterlauge trennen und waschen lässt, ohne eines Filters zu bedürfen. Das Waschen geschieht mit einer kalten, gesăttigten Lösung von Ammonsulfat. Man bringt den Alaun schliesslich mittelst eines Stuckchens Filtrirpapier in ein Tiegelchen und erhält durch Errhitzen uber dem Gebläse reine Thonerde. Die geringe Menge Mutterlauge nèbst der Waschflussigkeit wird in einem kleinen konischen Platintiegel zur Trockne gebracht und mit der nöthigen Vorsicht das Ammonsulfat zerstört. Der stark geglühte Inhalt des Tiegels giebt Tilansäure und Zirkonerde, deren Gewicht man bestimmt. Auf diese Weise gewonnen, befinden sich die Oxyde im Zustand feinster Zertheilung und lösen sich rasch und leicht in schmelzendem Phosphorsalze. Ich wäge davon auf der empfindlichen Kornwaage genau 2 Milligramm ab und löse das Pulver in einer schon gefertigten Phosphorsalzperle, deren Gewicht 0,065 beträgt $(0,12$ krystallisirtes Phosphorsalz). Um die abgewogene Menge vollständig in die Perle zu bringen, schüte ich dieselbe aus dem Waagenschälchen auf ein Papierscheibchen (schwedisches Filtrirpapier) von ca. $10^{\mathrm{mm}}$ Durchmesser, welches mit der letzten Spur der Substanz verbrannt wird; die verschwindend geringe Menge Asche ubt keinen störenden Einfluss. Nun wird die Perle etwa 2 Minuten in einer guten blauen Flamme reducirend behandelt (damit keine Kohlentheilchen beigemengt werden) und nachdem sie etwas erkaltet, in eine halbkugelförmige Porzellanschale scharf abgestossen. Bei einiger Uebung gelingt es leicht, stets dieselbe Menge des Glases abzustossen. In einer hermetisch geschlossenen Röhre bewahre ich 10 Perlen von gleichem Gewichle, jede 2 Milligramm Oxyd enthaltend von Mischungen reiner Titansäure und reiner Thonerde; so zwar, dass die Endglieder reiner Titansäure und reiner Thonerde entsprechen; die Zwischenglieder je 10 Prozente weniger Titansäure enthalten. 


\begin{tabular}{|c|c|c|}
\hline Also & $\mathrm{TiO}_{2}$ & $\mathrm{Al}_{2} \mathrm{O}_{3}$ \\
\hline & 100 & 0 \\
\hline & 90 & 10 \\
\hline & $\cdot$ & $\cdot$ \\
\hline & . & $:$ \\
\hline & 10 & 90 \\
\hline & 0 & 100 \\
\hline
\end{tabular}

Ausserdem bewahre ich 20 Mischungen, von 5 zu 5 Procent Titansäure, zum Gebrauche vorräthig. - Die Farbe der Perlen geht von tief röthlich violet, durch violetroth ins rosenrothe, indem die blauen Strablen bei abnehmender Sättigung mehr und mehr absorbirt werden. Ton und Tiefe der Farbe sind bei der Beurtheilung derselben zu berücksichtigen. Hat man durch Vergleich mit der Perlenscala die annähernde Lage der zu bestimmenden Perle festgestellt, so fertigt man nun die dazwischen fallende Perle der 5 prozentigen Stufenleiter, denn eine frische Perle ist, wenn auch nicht immer nothwendig, doch stets besser, insofern eine Art von freiwilliger Entglasung der lange aufbewahrten Perlen stattindet. - So gelingt es die relative Menge der Titansäure und Zirkonerde oder Thonerde bis auf 5 Prozente sicher zu bestimmen; bei mehrerer Uebung kann die Genauigkeit bis zu 2,5 gesteigert werden, ein mit keiner der vorhandenen Fällungsmethoden auch nur annähernd erreichbares Resultat.

Allerdings gehört eine ziemliche Geläufigkeit in der Handhabung des Löthrohres und ein gutes Auge dazu, was jedoch zu jeder colorimetrischen Methode auf nassem Wege ebenso unerlässlich ist. Man erkennt die Farbenunterschiede am besten, wenn man die Perlen auf eine weisse Unterlage bringt und das reflectirte Licht vergleicht. Im durchfallenden Lichte trilt die Tiefe des Tones weniger hervor. - Ich bin damit beschäftigt durch eine zweckmässig hergestellte Abstufung von Grün, womit die rothen Strahlen ausgelöscht werden, die Methode leichter sowohl als empfindlicher zu machen, jedoch ist dabei so Manches zu beobachten, um die Sache noch nicht spruchreif zu finden; immerhin aber mag die Methode, wie sie beschrieben wurde, meinen Mitarbeitern auf dem Felde der Mineralchemie, als Grundlage eigener Forschung dargeboten sein. -

Um nicht das Phosphorsalz für jede Perle abwägen zu müssen, bediene ich mich einer stets gleich grossen Oese ám Platindraht, schmelze daran Salz bis die erkaltete Perle (bei vertikaler Stellung der Oesenebene erkaltet, um die Perle symmetrisch zu erhalten) genau in ein dem Oesendurchmesser entsprechendes Eichmass passt. Durch 2 in einen Kork gesteckte Stecknadeln kann ein solches Kaliber leicht hergestellt werden.

In meiner Untersuchung des Titans habe ich viele hundert Perlen angefertigt und war von der Constanz der Farbenerscheinung in der Reduc- 
tionsflamme so betroffen, dass ich die Idee einer colorimetrischen Bestimmung erhielt. Ich wollte dieselbe nur als Hülfsmittel bei der Mineralbestimmung einführen, um rasch über die Natur einer Titanverbindung ins Klare zu kommen *).

Nur zwei chromatische Melalle - das Vanad und das Chrom - uben auf die Colorimetrie des Titans einen nachtheiligen Einfluss, beide färben namlich die Phosphorsalzperle in der Reductionsflamme grun. Da ihre Färbekraft nur von der des Kobaltes übertroffen wird, so wirken natürlicherweise schon geringe Mengen störend; denn roth und grün neutralisiren sich gegenseitig. Auffallendermassen scheint das Vanad die Titanverbindungen sehr häufig zu begleiten, denn ich fand in allen Titanmineralien von Magnet Gove Vanad, von Spuren bis zu 0.5 Prozent (Proceed. Acad. N. Sc. Philadelph. March 1876 j.

In dem sonst sehr reinen Rutil von Chester Co. Pa. fand ich kurzlich bei der Darstellung reiner Titansäure eine geringe Menge Chrom. Die Verwandtschaft der beiden Metalle ist offenbar bedeutend, denn die ganze Menge des Chroms schlug sich mit der Titansäure beim Kochen der schwefelsauren Lösung nieder, und wurde beim Schmelzen des Niederschlages mit Natroncarbonat und Nitrat erhalten. Durch diese Behandlung wird Vanad ebenfalls entfernt, und sollte man bei genauen Untersuchungen dieselbe stets ausfuhren.

\section{Arfvedsonit.}

Die Krystalle dieses Minerals zeigen sich als stark in die Länge gezogene sechsseitige Säulen mit den Winkeln der Hornblende. Mittelst des Anlegegoniometers wurde der stumpfe Säulenwinkel gleich $124{ }^{\circ} 30^{\prime}$ gefunden. Ein grosser Krystall, ca. $60^{\mathrm{mm}}$ lang und $25^{\mathrm{mm}}$ breil, zerbröckelte mit grosser Leichtigkeit unter dem Drucke der Finger in eine grosse Anzahl kleiner Prismen, welche nach der Behandlung mit heisser Salzsäure stark glänzende Flächen aufwiesen. Damit angestellte Messungen gaben für den slumpfen Winkel im Mittel 1240 $\mathrm{g}^{\prime}$.

Diesen Krystall erhielt ich lose, nicht im Gestein eingelagert. Die eingebetleten Individuen zeigten ein derartiges Verhalten nicht. Doch beobachtete ich an denselben neben der prismatischen Spaltungsrichtung auch noch eine dem Orthopinakoid parallel gehende. Die Enden der Säulen

*) In Magnet Cove, Arkansas, kommen Brookit, Perowskit, titanhaltiger Magnetit, tilanhaltiger Spinell und titanhaltiger Granat derb, oder so fein krystallinisch ausgebildet vor, dass eine physikalische Beslimmung unmöglich wird; darauf wollte ich die colorimetrische Methode zuerst verwenden und war von dem Resultat so befriedigt, dass ich die Anwendung auf die quantitalive Bestimmung bei der Analyse titanhaltiger Substanzen überhaupl, übertrug. 
erwiesen sich durchgehends abgebrochen, oder ganz unbestimmt ausgebildet. - Wegen der leichlen Zerbröckelung gelang es nicht eine optische Platte zu schleifen. Die Farbe des Minerales ist rabenschwarz (nach der Reinigung in Salzsäure), der Glanz halb metallisch, und der Strich lavendelblau. Die Härte gleich der des Orthoklases.

Volumgewicht $=3,433$ bei $12^{\circ} \mathrm{C}$. (mit grobem Pulver). Vor dem Löthrohre schmilzt das Mineral leicht zu einem schwarzen Glase und reagirt mit den Flüssen auf Kieselsäure, Eisen, Mangan und mit einiger Schwierigkeit erhält man auch die Titan reaktion im Phosphorsalze. Von kochender Salzund Schwefelsäure wird das Pulver kaum angegriffen und selbst unter Druck nur langsam aufgeschlossen.

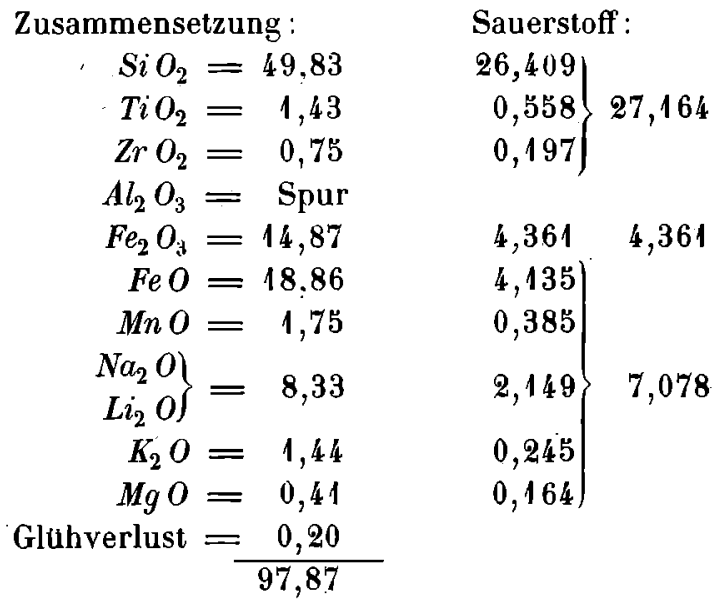

Hieraus resultiren die Atomverhältnisse :

$$
\begin{aligned}
& S i=0,847 \text { o : } 0,093=9,11 \text { oder abgerundet }=9 \\
& F e_{2}=0,093: "=1,00 " \text { " } "=1 \\
& F e=0,287: \text { : }=3,09 \text { » } \text { » }=3 \\
& N a=0,286: \text { : }=3,09 \text { » } \text { » }=3 \\
& O=2,431: \text { : }=26,1 \text { " } \quad=251 / 2
\end{aligned}
$$

Die geringen Procentsätze der übrigen Metalle sind in diese Zahlen mit ihren Aequivalenten eingerechnet.

Aus diesem Atomverhältniss folgt die empirische Formel :

$$
\mathrm{Na}_{6} \mathrm{Fe}_{6} \stackrel{\mathrm{III}}{\mathrm{Fe}_{4}} \mathrm{Si}_{18} \mathrm{O}_{51} \text {. }
$$

In wiefern das untersuchte Material homogen war, lässt sich nicht angeben, da eine mikroskopische Krilik nicht zur Anwendung gebracht werden konnte. Die Analyse wurde mit grosser Sorgfalt ausgeführt. Jedes Metall wurde zweimal bestimmt, die Alkalien sogar dreimal mit gleichbleibendem Resultate. Die Angabe des ferrosen Eisens beruht auf dem Ergebniss einer sehr gut gelungenen Aufschliessung nach der C o o k e'schen 
Methode (mit Schwefel- und Flusssäure), nachdem eine Bestimmung nach der Mi ts ch e rlich'schen Melhode ein nicht ganz genügendes Resultat geliefert hatte; bei letıterer war die Röhre eine Woche lang ununterbrochen auf $160^{\circ}$ erhalten worden und doch noch ein Bruchtheil der Substanz unzersetzt geblieben; dieser wurde mit der abgeschiedenen Kieselsäure nach der Titrirung mit Chamäleonlösung ausgewaschen, mit Natronlauge digerirt, der Ruckstand mit verdunnter Salzsäure gewaschen, dann geglüht und gewogen; er betrug etwa 10\%; hienach wurde die durch Titrirung gefundene Eisenoxydulmenge auf das Ganze berechnet und 17,95, also doch nahezu übereinstimmend mit der obigen Zahl, erhalten.

\section{Zirkon.}

Dieses Mineral findet sich in glänzenden, braunen bis schwarzen Krystallen, entweder im Quarze oder im Astrophyllit eingewachsen, von mikroskopischer Kleinheit bis $6^{\mathrm{mm}}$ Kantenlänge. Sie bilden stets die Combination (111) (110) (001), das Prisma erster Ordnung immer untergeordnet. Das im Allgemeinen an Zirkonen seltene basische Flächenpaar beobachtete ich an allen Individuen. An einem Krystalle fand ich eine rhombische Verzerrung der basischen Fläche und die Messung der Polkanten ergab in der That eine beträchtliche Winkeldifferenz, nämlich

\section{$120^{\circ} 56^{\prime}$ und $123^{\circ} 15^{\prime}$.}

Dieses abnorme Vorkommen veranlasste mich eine grössere Anzahl von Messungen vorzunehmen. Wenn auch scheinbar sehr glänzend, eignen sich die wenigsten Flächen zu genauen Messungen, da sie uneben sind. Ich fand jedoch alle Polkanten so nahezu gleich $123^{\circ}$ als die Beschaffenheit der Flächen dieses erwarten lässt. Der angeführte Fall von Verzerrung steht somit ganz vereinzelt da und ist wohl als eine Missbildung zu deuten. Wenn man die Krystalle längere Zeit mit starker Salzsäure kocht, so ändert sich die Farbe in ein fahles Rothgrau und die Flächen erweisen sich stellenweise sehr zerfressen. Volumgewicht bei $12{ }^{0} \mathrm{C} .=4,538$.

$$
\begin{aligned}
& \text { Zusammensetzung *): Atome: } \\
& \mathrm{SiO}_{2}=29,70 \quad \text { Si }=13,87 \quad 0,496 \\
& \mathrm{ZrO}_{2}=60,98 \quad \mathrm{Zr}=44,94 \quad 0,501 \\
& \mathrm{Fe}_{2} \mathrm{O}_{3}=9,20 \quad \mathrm{Fe}=6,44 \quad 0,057 \\
& M g O=0,30 \quad O^{-}=34,63 \quad 2,164
\end{aligned}
$$

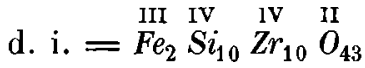

*) Das Pulver wurde vor der Zerlegung mit Salzsäure gekocht, bis kein Eisen mehr abgegeben wurde. Daraus und aus dem rationalen Atomverhältnisse darf man schliessen, dass das noch verbliebene Eisen einen Bestandtheil des Moleküls ausmacht. 


\section{Nachschrift : Die optischen Eigenschaften des Astrophyllit}

von H. Bückingr in Strassburg.

Auf Wunsch des Verf.'s der vorstehenden Abhandlungen, welcher ein Handstück mit Astrophyllit von Colorado an das min. Inst. der hiesigen Universität eingesandt hatte, unlernahm ich die optische Untersuchung desselben. Da die Beobachtungen von $\mathrm{Hintze}$ und $\mathrm{T}$ s $\mathrm{ch}$ ermak ergeben haben, dass sowohl Magnesia, als Kaliglimmer im mon os y m m et r i s chen Systeme krystallisiren, so galt es hauptsächlich, zu prüfen, ob die Mittellinie der optischen Axen, wie Des GI o i z e a x angiebt, normal zur Spaltungsfläche steht oder dagegen geneigt ist. Es ergab sich das Letztere unzweifelhaft, und es sind somit die krystallographischen Angaben Sche erer's, nach welchen die Krystalle als nach der Klinodiagonale verlängert monosymmetrisch $\mathrm{zu}$ betrachten sind, vollkommen bestätigt.

1. Astrophyllit von Brevig. Die optische Axenebene ist die Symmetrieebene; die zweite Mittellinie (in Oel; in Luft sind die Axen nicht sichtbar) bildet mit der Normalen zur Platte $3^{0} 30^{\prime}$ für Roth, $3^{0} 33^{\prime}$ für Gelb; es findet also fast gar keine Dispersion der Mittellinien statt. Diese Messung wurde nach der bekannten Methode, durch Spiegelung des Fadenkreuzes durch die Platte (s. P. Groth, physikal. Krystallographie, S. 485), ausgeführt; der höchstmögliche Fehler derselben, bedingt durch Krümmung der Platte, ergab sich bei deren goniomelrischer Untersuchung zu 37'. Die Neigung der Mittellinie findet genau in der Symmetrieebene, d. h. in der Längsrichtung der Spaltungslamellen statt. Der stumpfe Axenwinkel in Oel ist :

$$
2 H_{0}=122^{018^{\prime}}(\mathrm{Li}), \quad 124^{\circ} 52^{\prime}(\mathrm{Na}) \text {. }
$$

Der Pleochroismus ist deutlich : die Schwingungen parallel der Symmetrieaxe (b) zeigen Orange, diejenigen dazu senkrecht in der Spaltungsfläche und parallel der optischen Axenebene (c) Citrongelb.

2. Astrophyllit von Colorado. Derselbe besitzt ganz die gleichen Eigenschaften, dieselbe negative Doppelbrechung und dieselben Absorptionsverhältnisse. Die Neigung der Mittellinie gegen die Normale der Spaltungsfläche wurde an drei Platten gefunden zu $3^{\circ} 0^{\prime}, 2^{0} 42^{\prime}$ und $3^{\circ} 16^{\prime}$, bei höchstmöglichen Fehlern von $20-30^{\prime}$. Der Axenwinkel ist, wie auch bei demjenigen von Brevig, etwas schwankend. Es wurden Messungen an denselben drei Platten angestellt :

$\begin{array}{lccc} & L i: & N a & T l \\ \text { I. } 2 H_{0}=121038^{\prime} & 124^{\circ} 14^{\prime} & 125^{0} 6^{\prime} \\ \text { II. } & - & 126^{0} 49^{\prime} & - \\ \text { IIl. } & 12105^{\prime} & 124^{\circ} 28^{\prime} & -\end{array}$

